\title{
Endovascular treatment of hemodialysis-induced lower limb artery ischemia: retrospective analysis from a single center
}

\author{
Peng Wang ${ }^{1 *}$, Fei Yang ${ }^{1 *}$, Jing Yin ${ }^{1}$, Huajian Zhu ${ }^{2}$, Meng Zhang ${ }^{1}$, Caixiang Liu ${ }^{3}$ \\ ${ }^{1}$ Department of Interventional and Vascular Surgery, Affiliated Hospital of Jiangnan University, Wuxi, China; ${ }^{2}$ Department of Surgery of Traditional \\ Chinese Medicine, Affiliated Hospital of Jiangnan University, Wuxi, China; ${ }^{3}$ Department of Nephrology, Wuxi Affiliated Hospital of Nanjing \\ University of Traditional Chinese Medicine, Wuxi, China \\ Contributions: (I) Conception and design: C Liu, P Wang, F Yang; (II) Administrative support: C Liu; (III) Provision of study materials or patients: P \\ Wang, F Yang, J Yin, H Zhu, M Zhang; (IV) Collection and assembly of data: P Wang, F Yang; (V) Data analysis and interpretation: P Wang, F Yang; \\ (VI) Manuscript writing: All authors; (VII) Final approval of manuscript: All authors. \\ "These authors contributed equally to this work. \\ Correspondence to: Caixiang Liu. Department of Nephrology, Wuxi Affiliated Hospital of Nanjing University of Traditional Chinese Medicine, No. 8, \\ Zhongnan West Road, Wuxi 214071, China. Email: liucx1022@126.com.
}

Backgroundz Critical limb artery ischemia is one of common complications after hemodialysis, with endovascular therapy (EVT) having become its first-line treatment. There is no relevant study investigating the relationship between EVT and the prognosis of hemodialysis patients with critical lower limb ischemia, the most common site of vascular dysfunction.

Methods: This was a retrospective, nonrandomized, single-center study. Hemodialysis patients with critical lower limb ischemia between May 2015 and October 2018 were included in this study. Their demographic and clinical data and the results of laboratory test were collected. The outcomes included all-cause mortality, amputation, and revascularization. Kaplan-Meier analysis and log-rank test were used to assess overall survival and amputation-free survival. Univariable and multivariable hazard Cox regression analyses were performed to determine risk factors of amputation and mortality.

Results: In all, 67 hemodialysis patients were finally included in this study. The median age of included patients was $69.8 \pm 8.7$ years, and the median duration of hemodialysis was $44.1 \pm 9.2$ months. There was no significant difference between patients receiving and not receiving EVT in collected demographic and clinical data except for the duration of hemodialysis $(46.1 \pm 9.0$ vs. $41.7 \pm 9.0$ months; $\mathrm{P}=0.048)$. The level of high-density lipoprotein cholesterol (HDL-C) in patients receiving EVT was $1.4 \pm 0.6 \mathrm{mmol} / \mathrm{L}$, which was significantly lower than the $1.9 \pm 0.6 \mathrm{mmol} / \mathrm{L}$ in patients not receiving EVT $(\mathrm{P}<0.001)$. The results from the Kaplan-Meier curves indicated that the incidences of all-cause mortality and amputation were much lower in patients receiving EVT than in those not receiving EVT ( $\mathrm{P}=0.038$ and $\mathrm{P}=0.020)$. Hazard Cox regression analysis also indicated that $\mathrm{EVT}$ played protective role in all-cause mortality and amputation in hemodialysis patients with lower limb ischemia. Age, nutritional risk, stroke, and C-reactive protein (CRP) were also determined as independent risk factors of all-cause mortality according to multivariable analysis. Additionally, duration of hemodialysis and smoking history were identified as independent risk factors of amputation.

Conclusions: EVT could be an efficient treatment for critical lower limb ischemia in hemodialysis patients to reduce all-cause mortality and the incidence of amputation. Moreover, some risk factors, such as malnutritional and stroke, should be avoided to improve the prognosis of hemodialysis patients.

Keywords: Hemodialysis; lower limb artery ischemia; endovascular treatment; survival; amputation

Submitted Feb 23, 2021. Accepted for publication Apr 17, 2021.

doi: $10.21037 /$ apm-21-648

View this article at: http://dx.doi.org/10.21037/apm-21-648 


\section{Introduction}

The number of patients with end-stage renal disease has increased around the world over the past few decades (1). For these patients, regular hemodialysis is the most commonly used treatment. Hemodialysis is effective in controlling the symptoms of patients and preventing the deterioration of the disease. However, severe complications can occur during the treatment of hemodialysis in patients with endstage renal disease (2). Vascular dysfunction is one of the more common complications after hemodialysis. It usually results from severe calcification of vascular walls during hemodialysis due to the inability of hemodialysis to filter out the phosphorus and calcium (3). According to previous studies, the most common site of vascular dysfunction is the lower limbs, especially the below-knee arteries $(4,5)$. Critical limb ischemia is the most advanced form of vascular dysfunction. The occurrence of critical limb ischemia usually contributes to poorer prognosis and even a higher mortality of patients (6-8). There is thus an urgent need to develop effective treatment to reduce the incidence of critical limb ischemia and improve the quality of life in patients undergoing hemodialysis.

The current treatments of critical limb ischemia include palliative treatment, surgical bypass, and endovascular therapy (EVT). Surgical bypass can be performed for revascularization using artificial blood vessels or autologous veins (9). EVT usually uses balloons or stents to dilate calcified arteries and then relieves the symptoms of ischemia (10). Some studies have compared surgical bypass and EVT in hemodialysis patients, concluding that surgical bypass and EVT have comparable effectiveness in the treatment of critical limb ischemia $(11,12)$. However, distal bypass surgery is technically challenging due to severe vascular calcification in patients with diabetic nephropathy (13). Patients with organ dysfunction are also not suited to undergo surgical bypass, with these patients experiencing a high perioperative mortality of $20.4 \%$ (14). Therefore, EVT has gradually become the first-line treatment of critical limb ischemia in China due to its noninvasive nature.

Several previous studies have investigated the impacts of EVT on critical limb ischemia and confirmed its good efficacy $(10,15,16)$. Nevertheless, most of research has focused on ischemia in all limb types, and no specific study to determine the relationship between EVT and the prognosis of hemodialysis patients with critical lower limb ischemia has been conducted. As the most common type of limb ischemia, lower limb ischemia accounts for more than $50 \%$ of all limb ischemia $(10,17)$, and thus research regarding its treatments is of great significance. Here, we summarize the cases of hemodialysis patients with lower limb ischemia in our department and attempt to determine the effectiveness of EVT and the predictors of amputation and mortality in hemodialysis patients with lower limb ischemia. We present the following article in accordance with the STROBE reporting checklist (available at http:// dx.doi.org/10.21037/apm-21-648).

\section{Methods}

\section{Patient selection}

This was a retrospective, nonrandomized, and single-center study. Hemodialysis patients who were diagnosed as critical lower limb ischemia between May 2015 and October 2018 were included in this study. Patients who suffered from lower limb ischemia due to non-atherosclerotic diseases, such as embolic disease, autoimmune diseases, or trauma; those who suffered from severe psychiatric disorders; and those who had participated in other clinical trials were excluded from the study. According to the inclusion and exclusion criteria, 71 patients were finally enrolled, and their data were collected for further analysis. This study was approved by institutional ethical review board in Affiliated Hospital of Jiangnan University and conforms to the provisions of the Declaration of Helsinki (as revised in 2013). Individual consent for this retrospective analysis was waived.

\section{Data collection}

Patients who had any ischemic symptoms including chills, numbness, resting pain, skin ulcer, or gangrene; who had previous diagnosis and treatment; or who had an ankle-brachial index (ABI) score of less than 0.9 or a toebrachial index (TBI) less than 0.6, were defined as having lower limb ischemia (18). Their demographic and clinical data, including patient age, sex, duration of hemodialysis, nutritional risk (based on nutrition risk screening 2002 score $\geq 3$ ), smoking history, chronic comorbidities (including diabetes, hypertension, hyperlipidemia, coronary heart disease, cerebrovascular disease, and stroke), and Fontaine classification, were the collected by two independent investigators. Additionally, several laboratory test findings were also collected, including hemoglobin, platelets, highdensity lipoprotein cholesterol (HDL-C), low density 
lipoprotein cholesterol (LDL-C), albumin and C-reactive protein (CRP). The data collected by two investigators were verified by a third investigator, with any disagreement being resolved through discussion.

\section{EVT procedure}

The treatment of EVT in this study was performed by several skilled cardiologists. Arterial lesions were initially detected by selective artery angiography, and then EVT was performed in arteries with more than $75 \%$ stenosis or occlusions. Successful EVT procedures were defined as those with targeted arteries with less than $25 \%$ stenosis or occlusions and a straight blood flow from the aorta down to the ulcer or gangrene in the lower limbs according to angiography. Failed EVT procedure were defined as those with targeted arteries of more than $25 \%$ stenosis or occlusions in which the blood flow remained blocked at the ulcer or gangrene. Re-evaluation would be performed using angiography if some symptoms of lower limb ischemia occurred in EVT patients during the period of follow-up, with an additional revascularization procedure potentially being required. Antiplatelet drugs were routinely used in all patients receiving EVT.

\section{Outcomes}

The outcomes in this study included all-cause mortality, amputation, and revascularization. Patients were followed up by outpatient clinic, telephone, or internet every 1 or 2 months, and the median period of follow-up was 19 months (interquartile range, 15-27 months). Four patients were excluded from this study due to a relatively short period of follow-up of fewer than 6 months. The treatments of revascularization included surgical bypass or second EVT in patients showing symptoms of lower limb ischemia again after the treatment after EVT.

\section{Statistical analysis}

Variables in this study included categorical variables and continuous variables. Categorical variables are reported as numbers, while continuous variables are reported as mean \pm standard deviation (SD). Group differences were analyzed using chi-square tests for categorical variables or $t$ test for continuous variables when appropriate. KaplanMeier analysis and log-rank test were used to assess overall survival and amputation-free survival, and to further determine the effectiveness of EVT in the treatment of critical lower limb ischemia in hemodialysis patients. Univariable and multivariable hazard Cox regression analyses were then performed to determine the predictors of amputation and mortality in included patients. Statistical analysis in this study was performed using SPSS 20.0 (IBM Corp., Armonk, NY, USA) and Kaplan-Meier plots were curved using Graphpad prism 9.0.0 (GraphPad Software, San Diego, CA, USA).

\section{Results}

After excluding 4 patients with a short follow-up of less than 6 months, 67 patients were finally included in this study. Their demographic and clinical characteristics are listed in Table 1. The median age of included patients was $69.8 \pm 8.7$ years, and 35 of them were male patients. The median duration of hemodialysis in these patients was $44.1 \pm 9.2$ months. According to the Nutritional Risk Screening 2002 (NRS2002) scale, 40 patients were diagnosed with nutritional risk. The data of smoking history were also collected with 30 patients being identified as previous or current smokers. Most of included patients suffered from chronic comorbidities: 30 had diabetes, 39 had hypertension, 19 had hyperlipidemia, 29 had coronary heart disease, 9 had cerebrovascular disease, and 8 had stroke. We then defined the severity of lower limb ischemia using Fontaine classification, which revealed that 53 of 67 patients were diagnosed as stage IV.

Among the 67 included hemodialysis patients, 31 patients received EVT treatment and 36 patients did not receive EVT treatment (Table 1). Patients receiving EVT had a relatively shorter duration of hemodialysis than those not receiving EVT (46.1 \pm 9.0 vs.41.7 \pm 9.0 months, $\mathrm{P}=0.048)$. There was no significant difference between the 2 groups in other variables.

The results of laboratory test were also collected in this study, as shown in Table 2. Median levels of hemoglobin, platelets, HDL-C, LDL-C, albumin, and CRP were $99.5 \pm 17.0 \mathrm{~g} / \mathrm{L},(165.8 \pm 56.6) \times 10^{9} / \mathrm{L}, 1.7 \pm 0.6 \mathrm{mmol} / \mathrm{L}$, $2.4 \pm 1.2 \mathrm{mmol} / \mathrm{L}, 33.2 \pm 4.4 \mathrm{~g} / \mathrm{L}$, and $17.4 \pm 3.9 \mathrm{mg} / \mathrm{L}$ in all patients, respectively. The level of HDL-C in patients receiving EVT was $1.4 \pm 0.6 \mathrm{mmol} / \mathrm{L}$, which was significantly lower than the $1.9 \pm 0.6 \mathrm{mmol} / \mathrm{L}$ in patients not receiving EVT $(\mathrm{P}<0.001)$. Other results of laboratory test were similar between the two groups.

The outcomes in this study included all-cause mortality, amputation, and revascularization (Table 3). A total of 
Table 1 Demographic and clinical characteristics of hemodialysis patients with lower limb ischemia

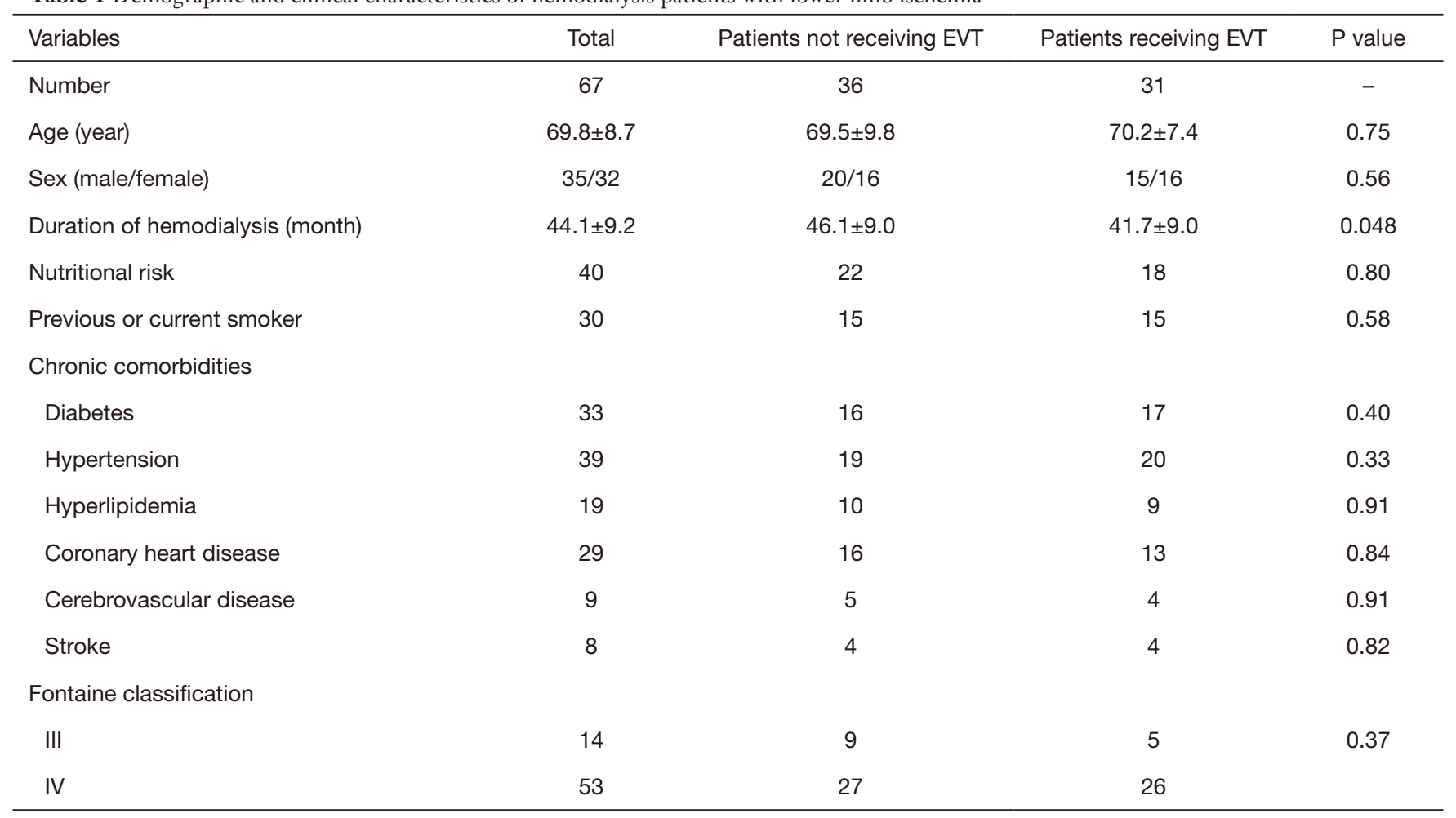

EVT, endovascular therapy.

41 patients $(61.2 \%)$ died within the follow-up period, including 27 patients (75\%) not receiving EVT and 14 patients (45.2\%) receiving EVT. Among all the included patients, 18 patients $(36.9 \%)$ underwent amputation: $14(38.9 \%)$ in the group of patients not receiving EVT and $4(12.9 \%)$ in the group of patients receiving EVT. The incidences of all-cause mortality and amputation were much lower in patients receiving EVT than in those not receiving $\operatorname{EVT}(\mathrm{P}=0.012$ and $\mathrm{P}=0.017)$. In addition, the total incidence of mortality or amputation was much lower in patients receiving EVT $(\mathrm{P}=0.002)$. There were 14 patients (45.2\%) who received EVT and required further treatment of revascularization. Kaplan-Meier curves were then generated to further compare the mortality and amputation in the two groups (Figure 1). The results from the KaplanMeier curves indicated that patients receiving EVT had an improved survival rate and a lower rate of amputation.

Cox hazard regression analysis was performed to determine the risk factors of mortality and amputation in hemodialysis patients with lower limb ischemia. As shown in Table 4, EVT, age, duration of hemodialysis, nutritional risk, diabetes, coronary heart disease, stroke, albumin, and CRP were identified as risk factors of mortality in included patients according to univariable analysis. After adjustment by multivariable analysis, EVT, age, nutritional risk, stroke, and CRP were determined as independent risk factors of mortality in hemodialysis patients with lower limb ischemia. Similarly, EVT, duration of hemodialysis, and previous or current smoker status were identified as independent risk factors of amputation according to multivariable analysis as shown in Table 5.

\section{Discussion}

This retrospective single-center study collected the data from 67 hemodialysis patients with lower limb ischemia and found that the treatment of EVT could reduce the incidence of amputation and all-cause mortality in these patients. To our knowledge, this is the first study in China to focus on the impact of EVT on critical lower limb ischemia. Based on the results of this study, the role of EVT in the treatment of hemodialysis patients with lower limb ischemia was further verified.

The all-cause mortality of $75 \%$ in our study was relatively higher than that reported in previous research $(19,20)$. This may be attributable to the fact that the 
Table 2 Laboratory tests of hemodialysis patients with lower limb ischemia

\begin{tabular}{|c|c|c|c|c|}
\hline Variables & Total & Patients not receiving EVT & Patients receiving EVT & $P$ value \\
\hline Platelets $\left(10^{9} / \mathrm{L}\right)$ & $165.8 \pm 56.6$ & $158.3 \pm 59.3$ & $170.2 \pm 53.6$ & 0.40 \\
\hline HDL-C (mmol/L) & $1.7 \pm 0.6$ & $1.9 \pm 0.6$ & $1.4 \pm 0.6$ & $<0.001$ \\
\hline LDL-C (mmol/L) & $2.4 \pm 1.2$ & $2.2 \pm 1.5$ & $2.6 \pm 1.1$ & 0.32 \\
\hline $\mathrm{CRP}(\mathrm{mg} / \mathrm{L})$ & $17.4 \pm 3.9$ & $17.2 \pm 3.7$ & $17.5 \pm 4.3$ & 0.75 \\
\hline
\end{tabular}

EVT, endovascular therapy; HDL-C, high-density lipoprotein cholesterol; LDL-C, low-density lipoprotein cholesterol; CRP, C-reactive protein.

Table 3 Outcomes of hemodialysis patients with lower limb ischemia

\begin{tabular}{lcccc}
\hline Variables & Total & Patients not receiving EVT & Patients receiving EVT & P value \\
\hline Number & 67 & 36 & 31 & 16 \\
Mortality or amputation & 47 & 31 & 27 & 0.002 \\
All-cause mortality & 41 & 14 & 4 & 0.012 \\
Amputation & 18 & - & 14 & 0.017 \\
Revascularization & 14 & - & 14 \\
\hline
\end{tabular}

EVT, endovascular therapy; HDL, high-density lipoprotein; LDL, low density lipoprotein; CRP, C-reactive protein.

majority of included patients in this study were at risk of malnutrition and family nursing care was insufficient. On the other hand, the incidence of amputation was similar to that in previous studies $(21,22)$, likely owing to adequate medication treatment and EVT.

There has been some controversy concerning surgical bypass and EVT over the past few decades. Generally, surgical bypass and EVT have been shown to have comparable effectiveness in the treatment of critical limb ischemia $(11,12)$. However, surgical bypass is more traumatic, which has led to the increased use of EVT in recent years. Kataoka et al. also reported that the incidences of all-cause death and amputation could be acceptable in hemodialysis patients with critical limb ischemia after receiving EVT (10). The study of Suematsu et al. found that the survival of hemodialysis patients remained poor after the treatment of EVT; however, the rate of limb salvage was relatively satisfactory (23). In our study, EVT showed a good therapeutic effect on the rate of amputation in these patients, but the all-cause mortality was also high, similar to that in Suematsu et al.'s study. Some effort has been made to further improve the effectiveness of EVT in hemodialysis patients. Ohtake et al. attempted to combine EVT with low-density lipoprotein apheresis to treat critical limb ischemia (18). Their findings indicated that the cumulative rate of major adverse limb events was significantly improved in the combined group compared to the EVT group. More studies should be performed to further verify the effectiveness of low-density lipoprotein apheresis in the treatment of critical limb ischemia.

Our study further identified severable variables as risk factors of mortality and amputation. For instance, similar to a previous study (23), older age was found to contribute to a higher mortality in the included patients. Nutritional status was also shown to be related to the mortality of hemodialysis patients, with malnutrition increasing the mortality of patients. NRS2002 scale was used in this study to assess the nutritional status; other scales used in previous research have also indicated a relationship between malnutrition and mortality in hemodialysis patients (18). CRP contributed to the mortality of included patients in our study and has been identified as an important risk factor of mortality in hemodialysis patients in previous work $(15,24)$. Moreover, smoking history was identified as independent predictor for major amputation in our study. It has been confirmed that smoking can lead 
A

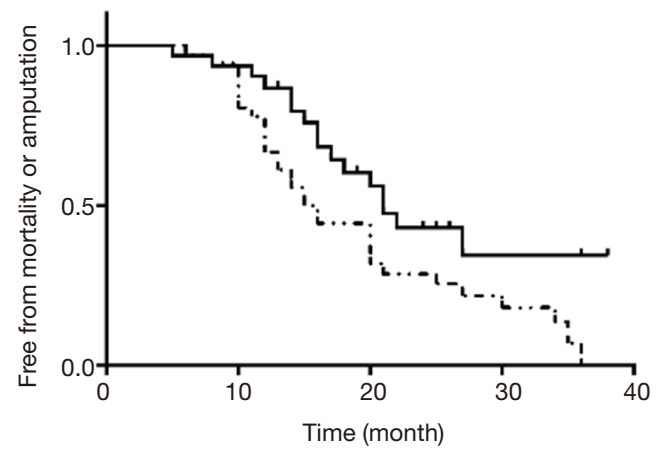

B

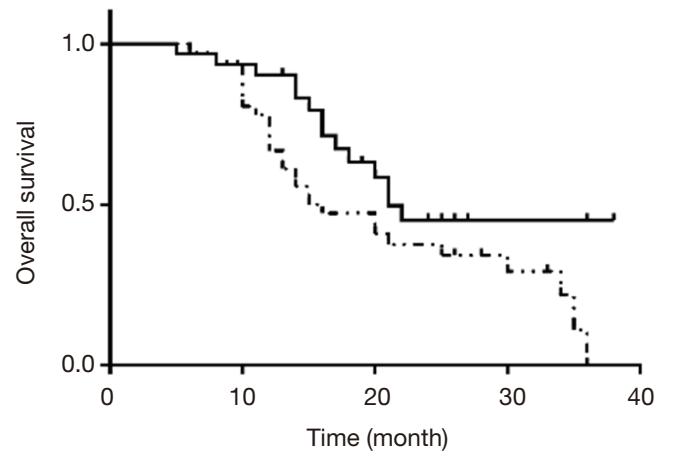

C

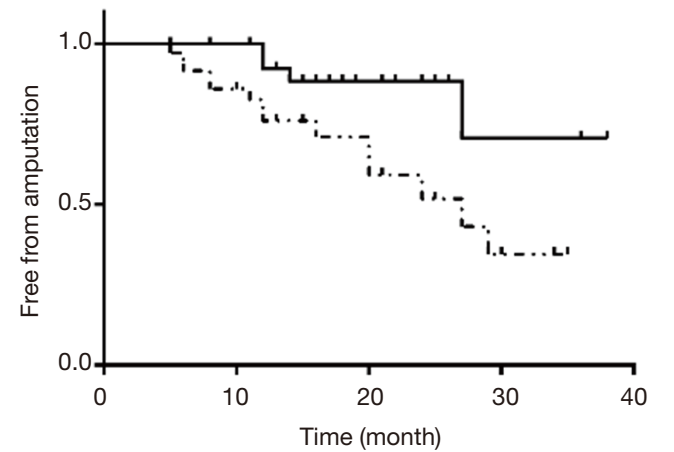

- '. Patients not receiving EVT

$\rightarrow$ Patients receiving EVT

Log-rank P value: 0.030

$\perp$ Patients receiving EVT

Log-rank P value: 0.038

- '. Patients not receiving EVT

工 Patients receiving EVT

Log-rank P value: 0.020

Figure 1 Kaplan-Meier curves of the mortality- and amputation-free rate among included patients. (A) Overall survival or amputation-free rate; (B) overall survival; (C) amputation-free rate.

to the recruitment of monocytes, induce the dysfunction of endothelial cells, and promote the development of thrombosis at damaged vessel walls (10).

Some limitations in this study should be mentioned. First, this study was performed from a single center and used a relatively small sample size. The low number of included patients hindered us from further analysis of the effects of EVT on lower limb ischemia. Also, multivariable regression analysis based on such a small sample size might have generated a degree of bias in this study. The second issue was that the treatment in patients not receiving EVT varied across each patient. Different treatments might have affected the prognosis of patients, but this study could not divide these patients into several subgroups considering the sample size of the included patients. Finally, this was a retrospective study, and retrospective data collection might have produced discrepant data. We are therefore planning to conduct a 
Table 4 Independent risk factors for all-cause mortality in hemodialysis patients with lower limb ischemia

\begin{tabular}{lcc}
\hline Variables ${ }^{*}$ & HR (95\% Cl) & P value \\
\hline EVT & $0.101(0.023-0.452)$ & 0.003 \\
Age & $1.015(1.008-1.023)$ & 0.031 \\
Duration of hemodialysis & $0.972(0.926-1.020)$ & 0.255 \\
Nutritional risk & $7.594(2.365-24.383)$ & 0.001 \\
Diabetes & $2.660(0.429-16.483)$ & 0.293 \\
Coronary heart disease & $4.033(0.407-39.998)$ & 0.234 \\
Stroke & $4.267(1.338-13.608)$ & 0.027 \\
Albumin & $1.054(0.950-1.170)$ & 0.322 \\
CRP & $1.006(1.001-1.013)$ & 0.045 \\
\hline
\end{tabular}

*, multivariate Cox hazard regression analysis included EVT, age, duration of hemodialysis, nutritional risk, diabetes, coronary heart disease, stroke, albumin, and CRP as covariates with $\mathrm{P}<0.05$ by univariate analysis. Italic values indicate significant difference. EVT, endovascular therapy; $\mathrm{HR}$, hazard ratio; $\mathrm{Cl}$, confidence interval; $\mathrm{CRP}, \mathrm{C}$-reactive protein.

Table 5 Independent risk factors for amputation in hemodialysis patients with lower limb ischemia

\begin{tabular}{lcc}
\hline Variables $^{*}$ & HR (95\% Cl) & P value \\
\hline EVT & $0.276(0.090-0.840)$ & 0.023 \\
Age & $1.031(0.976-1.088)$ & 0.274 \\
Duration of hemodialysis & $2.295(1.012-5.208)$ & 0.047 \\
Diabetes & $2.581(0.421-15.830)$ & 0.306 \\
Previous or current smoker & $3.932(1.414-10.934)$ & 0.009 \\
Hyperlipidemia & $3.803(0.152-95.111)$ & 0.416 \\
CRP & $3.172(0.929-10.833)$ & 0.065 \\
\hline
\end{tabular}

*, multivariate Cox hazard regression analysis included EVT, age, duration of hemodialysis, diabetes, previous or current smoker status, hyperlipidemia, and CRP as covariates with $\mathrm{P}<0.05$ by univariate analysis. Italic values indicate significant difference. EVT, endovascular therapy; HR, hazard ratio; Cl, confidence interval; CRP, C-reactive protein.

prospective study in the future.

\section{Conclusions}

This retrospective single-center study included 67 hemodialysis patients with critical lower limb ischemia and found EVT to be an efficacious treatment for lower limb ischemia in reducing all-cause mortality and the incidence of amputation. Furthermore, variables such as older age, nutritional risk, stroke, and high level of CRP were determined to be independent risk factors of mortality in hemodialysis patients. Longer duration of hemodialysis and smoking history also acted as risk factors of amputation in included patients.

\section{Acknowledgments}

Funding: This work was supported by the Wuxi Key Medical Talent Fund for Young People in "Strengthening Health By Science and Education" (Wuxi Health and Family Planning Commission; No. QNRC070 and 2017-2020).

\section{Footnote}

Reporting Checklist: The authors have completed the STROBE reporting checklist. Available at http://dx.doi. org/10.21037/apm-21-648

Data Sharing Statement: Available at http://dx.doi. 
org/10.21037/apm-21-648

Conflicts of Interest: All authors have completed the ICMJE uniform disclosure form (available at http://dx.doi. org/10.21037/apm-21-648). The authors have no conflicts of interest to declare.

Ethical Statement: The authors are accountable for all aspects of the work in ensuring that questions related to the accuracy or integrity of any part of the work are appropriately investigated and resolved. This study was approved by institutional ethical review board in Affiliated Hospital of Jiangnan University and conforms to the provisions of the Declaration of Helsinki (as revised in 2013). Individual consent for this retrospective analysis was waived.

Open Access Statement: This is an Open Access article distributed in accordance with the Creative Commons Attribution-NonCommercial-NoDerivs 4.0 International License (CC BY-NC-ND 4.0), which permits the noncommercial replication and distribution of the article with the strict proviso that no changes or edits are made and the original work is properly cited (including links to both the formal publication through the relevant DOI and the license). See: https://creativecommons.org/licenses/by-nc-nd/4.0/.

\section{References}

1. Wei SY, Huang JC, Chen SC, et al. Unequal Arterial Stiffness With Overall and Cardiovascular Mortality in Patients Receiving Hemodialysis. Am J Med Sci 2016;351:187-93.

2. Sági B, Peti A, Lakatos O, et al. Pro- and antiinflammatory factors, vascular stiffness and outcomes in chronic hemodialysis patients. Physiol Int 2020. [Epub ahead of print]. doi: 10.1556/2060.2020.00026.

3. Honda Y, Hirano K, Yamawaki M, et al. Wound healing of critical limb ischemia with tissue loss in patients on hemodialysis. Vascular 2017;25:272-82.

4. Ohtake T, Oka M, Ikee R, et al. Impact of lower limbs' arterial calcification on the prevalence and severity of PAD in patients on hemodialysis. J Vasc Surg 2011;53:676-83.

5. Iida O, Soga Y, Kawasaki D, et al. Angiographic restenosis and its clinical impact after infrapopliteal angioplasty. Eur J Vasc Endovasc Surg 2012;44:425-31.

6. Hou JS, Wang CH, Lai YH, et al. Serum Malondialdehyde-Modified Low-Density Lipoprotein Is a
Risk Factor for Central Arterial Stiffness in Maintenance Hemodialysis Patients. Nutrients 2020;12:2160.

7. Jiao D, Guo F, Yue M, et al. Ischemia-Modified Albumin Is Associated with Arterial Stiffness in Hemodialysis Patients. Int Heart J 2020;61:332-7.

8. Tokuda T, Hirano K, Sakamoto Y, et al. Use of the Wound, Ischemia, foot Infection classification system in hemodialysis patients after endovascular treatment for critical limb ischemia. J Vasc Surg 2018;67:1762-8.

9. Gu YQ, Zhang J, Qi LX, et al. Surgical treatment of 82 patients with diabetic lower limb ischemia by distal arterial bypass. Chin Med J (Engl) 2007;120:106-9.

10. Kataoka S, Yamaguchi J, Nakao M, et al. Clinical outcome and its predictors in hemodialysis patients with critical limb ischemia undergoing endovascular therapy. J Interv Cardiol 2017;30:374-81.

11. Shiraki T, Iida O, Takahara M, et al. Comparison of Clinical Outcomes after Surgical and Endovascular Revascularization in Hemodialysis Patients with Critical Limb Ischemia. J Atheroscler Thromb 2017;24:621-9.

12. Ito R, Kumada $Y$, Ishii $H$, et al. Clinical Outcomes after Isolated Infrapopliteal Revascularization in Hemodialysis Patients with Critical Limb Ischemia: Endovascular Therapy versus Bypass Surgery. J Atheroscler Thromb 2018;25:799-807.

13. Aiello A, Anichini R, Brocco E, et al. Treatment of peripheral arterial disease in diabetes: a consensus of the Italian Societies of Diabetes (SID, AMD), Radiology (SIRM) and Vascular Endovascular Surgery (SICVE). Nutr Metab Cardiovasc Dis 2014;24:355-69.

14. Biancari F, Salenius JP, Heikkinen M, et al. Risk-scoring method for prediction of 30-day postoperative outcome after infrainguinal surgical revascularization for critical lower-limb ischemia: a Finnvasc registry study. World J Surg 2007;31:217-25; discussion 226-7.

15. Abualhin M, Gargiulo M, Bianchini Massoni C, et al. A prognostic score for clinical success after revascularization of critical limb ischemia in hemodialysis patients. J Vasc Surg 2019;70:901-12.

16. Odajima $S$, Inoue $T$, Hamana $T$, et al. Skin ulcer due to hemodialysis access-induced distal ischemia treated with arteriovenous fistula banding and endovascular therapy. J Cardiol Cases 2019;20:155-7.

17. Popp W, Knoll F, Sprenger-Mahr H, et al. Alprostadil treatment of critical limb ischemia in hemodialysis patients : A retrospective single-center analysis. Wien Klin Wochenschr 2019;131:209-15.

18. Ohtake T, Mochida Y, Matsumi J, et al. Beneficial Effect 
of Endovascular Therapy and Low-Density Lipoprotein Apheresis Combined Treatment in Hemodialysis Patients With Critical Limb Ischemia due to Below-Knee Arterial Lesions. Ther Apher Dial 2016;20:661-7.

19. Yorifuji M, Kuragano T, Kawada S, et al. Factors associated with serum magnesium and vascular stiffness in maintenance hemodialysis patients. Hemodial Int 2018;22:342-50.

20. Teixeira G, Almeida P, Loureiro L, et al. Arterial percutaneous angioplasty in hemodialysis access: Endovascular treatment of hand ischemia. J Vasc Access 2020. [Epub ahead of print]. doi: 10.1177/1129729820946654.

21. Poulin A, Bellemare PL, Fortier C, et al. Acute effects of cinacalcet on arterial stiffness and ventricular function in hemodialysis patients: A randomized double-blinded crossover study. Medicine (Baltimore) 2017;96:e6912.

Cite this article as: Wang P, Yang F, Yin J, Zhu H, Zhang M, Liu C. Endovascular treatment of hemodialysis-induced lower limb artery ischemia: retrospective analysis from a single center. Ann Palliat Med 2021;10(4):4661-4669. doi: 10.21037/apm-21-648
22. Nakano M, Hirano K, Yamauchi Y, et al. Three-year clinical outcome after infrapopliteal angioplasty for critical limb ischemia in hemodialysis patients with minor or major tissue loss. Catheter Cardiovasc Interv 2015;86:289-98.

23. Suematsu N, Iida O, Takahara M, et al. Prognostic Factors in Hemodialysis Patients Undergoing Endovascular Treatment for Critical Limb Ischemia due to Isolated Below-the-Knee Disease. J Atheroscler Thromb 2015;22:404-14.

24. Tokuda T, Oba Y, Koshida R, et al. Validation of Global Limb Anatomical Staging System (GLASS) in patients with hemodialysis and Chronic Limb-Threatening Ischemia after endovascular treatment. Heart Vessels 2021. [Epub ahead of print]. doi: 10.1007/s00380-020-01747-1.

(English Language Editor: J. Gray) 